\title{
PENSADORES Y CIENTÍFICOS EN LA ESPAÑA DE LOS SIGLOS XIX Y XX
}

\section{THINKERS AND SCIENTISTS OF SPAIN IN THE 19th AND 20th CENTURY}

\author{
Adela Roldán Márquez \\ Miriam González Limón \\ Universidad de Sevilla. España/Spain \\ roldan@us.es \\ miryam@,us.es
}

Recibido/Received: 20/05/2010

Aceptado/Accepted: 27/07/2010

\section{RESUMEN}

En España se produce un desarrollo del pensamiento económico tardío, comparable al que tiene lugar en otras ciencias sociales, por diversos factores históricos que llevaron a situarlo en los márgenes de la sociedad y que obligaron a muchos de sus protagonistas al exilio.

PALABRAS CLAVE

Antiguo Régimen, modernización, conservadurismo, liberalismo, guerra civil.

\section{ABSTRACT}

The development of economic thought in Spain takes place later than in other societies, in a similar way to what happened with the rest of the social sciences, due to different historical factors that placed it in the margins of society and drew many of its most important representatives out to exile.

\section{KEYWORDS}

Old Regime, modernization, conservatism, liberalism, civil war.

\section{INTRODUCCIÓN}

Una de las consecuencias de que en España no tuvieran lugar ni una revolución industrial ni una revolución política es que su historia intelectual, deformada continuamente por el conservadurismo (predominante en la sociedad durante prolongados periodos de tiempo) y la censura (impuesta por aquellos que detentaban el poder político y que, de una $u$ otra manera, imponían su visión y su versión de la realidad), ha impedido no solamente que los españoles conozcamos y nos beneficiemos de importantes pensadores en muy distintos ámbitos de la ciencias sociales, sino que el devenir económico y social del propio país se 
beneficiara de sus aportaciones y conocimientos. Muchos de esos pensadores, excluidos de los foros del conocimiento y de las posiciones influyentes, hubieron de exilarse.

Este exilio político, clara muestra de la general incapacidad española para admitir la disidencia ideológica, tuvo una amplísima repercusión sobre la vida intelectual de nuestro país fundamentalmente en tres momentos. Los dos primeros tuvieron lugar durante el primer tercio del siglo XIX, periodo en el que se produjeron dos grandes oleadas de exiliados: la primera, al finalizar la Guerra de Independencia que tuvo como principales protagonistas a los afrancesados políticos ya que cuando en 1813 abandona España José Bonaparte le acompañaron unas 12 o 15.000 personas de las que más de 4.000, que habían ocupado cargos durante la invasión, no pudieron siquiera regresar en 1814 tras el decreto del mes de mayo dictado por Fernando VII; la segunda, tras la reinstauración del absolutismo fernandino después del trienio liberal 1820-1823, fue protagonizada por los liberales. El tercer momento ocurrió ya en el siglo XX, fundamentalmente a partir del 1 de abril de 1939, cuando se pone fin a la Guerra Civil y comienza el denominado por el franquismo "tiempo de la victoria". Ese año, aquellos que habían perdido la guerra se vieron en la disyuntiva de quedarse y sufrir la dura represión de la dictadura o huir de ella con el objetivo de salvar la vida y, para un buen número de ellos, preservar la esperanza y el ideal de restituir la República. Esta esperanza venía motivada por el contexto internacional ya que, pocos meses después del fin de la guerra española, en el mes de septiembre se declararía la II Guerra Mundial y para muchos de los exiliados esta guerra no suponía solamente la resistencia militar sino que tenía unas dimensiones mundiales en las que la cultura, con la que se hallaban comprometidos, se consideraba un arma más de lucha.

\section{I}

Los intelectuales exilados del siglo XIX tuvieron como destino principal Europa, los del siglo XX América. En el primer periodo, la inmensa mayoría de los exiliados emigró a Francia, de donde fueron regresando paulatinamente, sobre todo a partir de 1820. En el segundo, los exiliados se establecieron mayoritariamente en Inglaterra, aunque muchos de ellos, tras la revolución de 1830, se trasladaron a la isla de Jersey, cerca de la costa francesa. Allí permanecieron hasta que en 1834 se produjo la amnistía que permitió su regreso. En el tercero, los más fueron a México.

En todos los casos, miles de ciudadanos españoles se vieron obligados a marcharse del país, aunque la incidencia mayor en la vida española se produjo, no por la sangría cuantitativa que supusieron dichos exilios, sino por la categoría intelectual de los propios exiliados.

Así, entre los afrancesados exiliados figuraban altos funcionarios, literatos, profesionales y hombres de ciencia. Entre las figuras intelectuales más distinguidas se encontraban Leandro Fernández de Moratín, José Antonio Meléndez Valdés, Juan Sempere Guarinos, José Marchena, Alberto Lista, Juan Antonio Llorente, Antonio Ortiz de Zárate, Manuel Silvela, etc.

En lo relativo al exilio liberal de los años veinte, la práctica totalidad de la elite intelectual española de la época se vio obligada a emigrar: Ángel Saavedra, José de Espronceda, Antonio Alcalá Galiano, Agustín Argüelles, Juan Álvarez Mendizábal, Álvaro Flórez Estrada, José Canga Argüelles, los hermanos Jaime y Joaquín Lorenzo de Villanueva, Telesforo de Trueba y Cossío, Vicente Salvá, etc. Con este proceso de exclusión y el 
consiguiente exilio, mientras que en nuestro país el panorama intelectual se asemejaba a un improductivo erial, Londres se convertía en el centro cultural de España e Hispanoamérica.

De lo arraigada que está esta tradición de exclusión y exilio en la historia española daba cuenta ya Marcelino Menéndez Pelayo en su Historia de los heterodoxos españoles que, publicada en tres volúmenes entre 1880 y 1882, tuvo un gran éxito al editarse de la misma 4000 ejemplares, elevado número para la época, que se agotaron con gran rapidez.

La explicación del exilio en estos dos primeros momentos es que si a comienzos del siglo XIX el intento de crear un Estado moderno es guiado por los liberales, que aprovechan la invasión francesa para presentar lo que era una guerra dinástica como una guerra de independencia nacional y asentar las bases de un moderno Estado nacional centralizado, empeño también asumido por los afrancesados y los llamados conservadores, el siglo finaliza con los conservadores bien asentados, el liberalismo en retroceso y el nacionalismo español ligado las más de las veces al pensamiento reaccionario y por ello siempre cuestionado. Es más, muchas veces la falta de la existencia de un verdadero sentimiento nacional se utiliza para explicar el hecho que a finales del siglo XIX en España lo característico fuese que los jóvenes se embarcaran en proyectos tales como el internacionalismo proletario o las identidades nacionales que rivalizaban con la española, especialmente el catalanismo en el primer tercio del siglo XX. Pero, a pesar de todo ello, fueron los conservadores, muchos de ellos afrancesados, los que sentaron las bases para el desarrollo del Estado cuando finaliza la I Guerra Carlista.

El carlismo representa sobre todo el medio rural, la defensa del patrimonio comunal y la contrarrevolución y el extremismo apostólico, es un movimiento antiliberal y siempre, durante las tres guerras carlistas (1833-1840, 1846-1849, 1872-1876) estuvo lastrado no solamente por esa contrarrevolución sino también por la aversión del campo contra la ciudad característico del País Vasco y de otros lugares como, por ejemplo, Cataluña. A esto se unió el desigual desarrollo español que condujo a un proceso de modernización cultural desequilibrado que giraba sobre las dos únicas ciudades (Bilbao y Barcelona) que podían competir con la capital, lo que supuso la aparición de graves tensiones por la no correspondencia entre el poder político (ejercido por las elites que provenían de Castilla y de Andalucía) y el económico. Pero esto no tuvo como efecto que las elites periféricas lideraran la creación de un Estado liberal modernizador sino que estas elites se caracterizaron, en general, por su apoyo al carlismo antimodernizante.

No hay que olvidar que la fecha en la que en España terminó la Edad Moderna y con ella el Antiguo Régimen, comenzando otra época que el mundo académico latino llama "contemporánea", es 1808 cuando, con la Guerra de la Independencia, se comienza la construcción de la identidad nacional y que a partir de aquel momento se pudo empezar a hablar del nacionalismo español en el sentido contemporáneo del término. Siguiendo a José Álvarez Junco esta fecha marca el momento de ruptura en que el patriotismo pasó a ser plenamente nacional, al menos entre las elites. Para Álvarez Junco es justamente en el transcurso de la guerra antinapoleónica y fue obra de los liberales. Reunidas en Cádiz las Cortes, institución que no se había reunido salvo con funciones protocolarias desde hacía siglos, las elites modernizadoras aprovecharon aquella ocasión para intentar imponer un programa de cambios sociales y políticos. Y la manera de defender la competencia de aquel organismo para tal función reformadora consistió en lanzar la idea revolucionaria de la nación como titular de la soberanía en el momento en que faltaba el monarca. El mito nacional fue aceptado por los demás como el ancla de salvación en aquellas difíciles circunstancias. Gracias a ese planteamiento nacional se deslegitimó al ejército napoleónico, como extranjero y tiránico, y se desprestigió a los colaboradores de José Bonaparte de un 
plumazo al llamarlos "afrancesados", es decir, no españoles. Era preciso inventar un mito político creíble y de suficiente potencia como para rivalizar con el sacralizado monarca y en España, siguiendo a Francia, se inventó la nación. Era el artilugio que permitía liquidar la legitimidad regia y, con ella, todos los privilegios heredados.

El planteamiento era que la abdicación de los Borbones en Napoleón era inadmisible ya que el dominio de los Bonaparte a través de José habría requerido el consentimiento de la nación. Así, el planteamiento liberal de la guerra consistió en convertir lo que en principio era un repudio al "tirano" Bonaparte en una toma de posición contra la "tiranía" como principio, es decir, contra cualquier persona, extranjera o española, que pretendiera tomar decisiones políticas sin tener en cuenta la voluntad de "la nación". La rebelión contra los franceses era pues una lucha por la "libertad".

Los diputados de las Cortes de Cádiz aprobaron los célebres artículos segundo y tercero de la Constitución: "la nación española es libre e independiente y no es ni puede ser patrimonio de ninguna persona ni familia" y "la soberanía reside esencialmente en la nación y por lo mismo pertenece a ésta exclusivamente el derecho de establecer sus leyes fundamentales". Todo aquel proceso político y cultural fue, en realidad, cosa de elites políticas e intelectuales, con muy reducido alcance o impacto popular. No hay motivos para pensar que antes de la sublevación de 1808 las nuevas ideas nacionalistas hubieran rebasado los selectos círculos políticos y literarios cercanos a la corte y se hubieran difundido entre la gran mayoría de la población, aislada por el ruralismo y el analfabetismo. Pero las elites impulsoras de aquella nueva forma de identificación mostraban escaso interés por expandir tales ideas y sentimientos en los medios populares. El pueblo, por definición, no entendía de esas cosas; y mejor sería que no pretendiera entenderlos.

El giro populista de los liberales a partir de la Guerra de la Independencia representó un cambio tan radical respecto a las representaciones del mundo mental anterior que toda persona educada en aquel debió de pensar que el nuevo discurso era cosa de locos. No fue fácil, desde luego, cambiar de manera tan completa la manera de pensar de las elites en relación con el pueblo. Pero los liberales aceptaron y difundieron el mito del pueblo como el luchador heroico por la libertad nacional ya que de él se derivaba la consecuencia política que buscaban: el derecho a participar en la toma de decisiones que afectaran a la colectividad. Por la misma razón, los absolutistas mostraban un entusiasmo casi exactamente opuesto al de los liberales ante la intervención popular. En cierto modo, era una falta de visión política, porque la idea del "pueblo" podría haberse vinculado a los valores del Antiguo Régimen, pero los conservadores tendieron a mantener, en los años de la guerra contra Napoleón, los viejos temores al pueblo.

Que la guerra de 1808-1814 tuviera un carácter tan popular y tan espontáneo, y sobre todo que estuviera tan inspirada por sentimientos patrióticos, es algo sobre lo que los historiadores actuales no sienten tanta seguridad como los del XIX. Pero otros autores, como por ejemplo Carmen Iglesias, mantienen la veracidad y la importancia de que, ante el vacío de poder realmente existente, el Ejército y el pueblo cogieran las riendas contra la invasión, que no consideran, en absoluto, una guerra civil a pesar a ser una lucha entre dinastías y similar por ello a las guerras carlistas. Otros más mantienen que el 2 de Mayo fue obra del populacho con Ruiz, Daoiz y Velarde. Pero lo que realmente ocurriera no importa. Lo importante es lo que la gente creyó que había ocurrido. Y la "Guerra de la Independencia" quedó marcada de forma indeleble con rasgos populistas. No obstante, aquella confianza en el pueblo por parte de las elites liberales puede que no pasara de ser sino un equívoco que 
diera lugar a las muchas decepciones que vinieron después. Porque realmente el proceso de nacionalización desarrollado en España durante aquel siglo no fue radicalmente distinto a la construcción de la identidad durante el Antiguo Régimen: siempre giró en torno a construcciones culturales, y con lealtades elaboradas alrededor de ellas, que fueron interiorizadas por las elites y que apenas se difundieron entre las capas populares, ya que el aislamiento entre unas y otras no dejó de existir a lo largo del XIX, e incluían la consideración del adversario político como enemigo. Lo único nuevo a partir de dicha guerra fue un giro retórico: la veneración nominal hacia el pueblo como último baluarte de las libertades y los sentimientos patrios.

Pero además, para entender la exclusión y el exilio, no hay que olvidar que la legitimación de la violencia en tanto que fuerza que se ejerce sobre otro para imponer la voluntad propia, una particular visión del orden social o del Estado al conjunto de la sociedad, ha sido habitual en España desde la revolución liberal de los años treinta del siglo XIX hasta la transición a la democracia después del periodo franquista. Durante todo ese tiempo, según Santos Juliá, la violencia así concebida fue defendida en teoría y puesta en práctica por formaciones políticas, fuerzas sociales y burocracias del Estado que gozaron de gran apoyo social durante dilatados periodos de tiempo como, por ejemplo, los carlistas, los anarquistas, los socialistas, los monárquicos, los católicos, los fascistas, los patronos, los militares y los nacionalistas. Esto explica la profusión de guerras internas, civiles o no, desde las llamadas carlistas del XIX hasta la civil de 1936. Papel importante en ello tiene el que las guerras degeneraran en movimiento populares que no se ajustaban a las normas de las mismas, lo que fue frecuente, como narra Caro Baroja, entre los que inicialmente eran considerados carlistas.

En cualquier caso, tanto si se mitificó como si no, el éxito de la Guerra de la Independencia se convirtió en un problema porque tras de ella nadie educó al pueblo en los nuevos ideales, pero los ideales adquirieron vida propia y el pueblo quedó desligado del proyecto constitucionalista y modernizador. De ahí la depresión generalizada entre las clases medias cultas en 1898, cuando llegaron las noticias del hundimiento definitivo de las colonias y el imperio y comprobaron que, sin embargo, el pueblo seguía yendo a los toros, como si nada hubiera ocurrido. Aún entonces Azorín, Baroja, Marquina y otros intelectuales se sumaron a un homenaje "Al pueblo" por sus sacrificios durante la guerra de Cuba.

De este exilio del siglo XIX, cuando buena parte de los españoles intentan sustituir al Antiguo Régimen por un Estado moderno del tipo liberal burgués, podemos destacar la figura de Álvaro Flórez Estrada, un perfecto desconocido para la España oficial hasta bien entrada la segunda mitad del siglo XX. Nacido en 1776 e hijo de un "ilustrado", recibió una educación europea licenciándose en Leyes. Bajo el reinado de Carlos IV fue desterrado en 1794 por Godoy, quien se alió en 1796 con Napoleón y se oponía a la influencia de la Revolución Francesa. Fue Flórez, defensor de la libertad de imprenta, quien realizó un proyecto de Constitución política liberal y quien realiza una Constitución para la Nación española, presentada a S. M. la Junta Suprema Gubernativa de España e Indias en 1 de noviembre de 1809 que publicó en Inglaterra, al igual que otras muchas de sus obras, al no estar aprobada en Cortes la libertad de imprenta. Y en Inglaterra se exila cuando Fernando VII comienza la primera represión reaccionaria del siglo XIX que le condenaría a muerte. Allí inició sus estudios económicos de los clásicos ingleses de la economía política. Volvió a España en 1820 con el triunfo del levantamiento liberal de Riego y cuando en 1823, tras la ejecución de Riego aún bajo el reinado de Fernando VII, de nuevo la reacción vuelve a condenarlo a muerte, vuelve a Inglaterra donde permanece estudiando economía hasta 1830, fecha en que se traslada a París y donde permanece hasta que vuelve definitivamente a 
España en 1834 con la "amnistía cristiana" y donde muere en 1853. En España en 1833, a la muerte de Fernando VII y con la regencia de M $^{\text {a }}$ Cristina, para vencer a los carlistas se busca la alianza con los liberales y se promulga una amnistía que se amplía al año siguiente cuando se promulga el Estatuto Real que sustituye el absolutismo con una constitución liberal que permite la existencia de una monarquía liberal de carácter moderado y burgués vigente ya durante la mayor parte del siglo.

Otras figuras intelectuales del exilio del siglo XIX fueron, en su primera época, Leandro Fernández de Moratín, José Antonio Meléndez Valdés, Juan Sempere Guarinos, José Marchena, Alberto Lista, Juan Antonio Llorente, Antonio Ortiz de Zárate, Manuel Silvela, etc. En lo relativo al exilio liberal de los años veinte, la práctica totalidad de la elite intelectual española de la época se vio obligada a emigrar: Ángel Saavedra, José de Espronceda, Antonio Alcalá Galiano, Agustín Argüelles, Juan Álvarez Mendizábal, José Canga Argüelles, los hermanos Jaime y Joaquín Lorenzo de Villanueva, Telesforo de Trueba y Cossío, Vicente Salvá, etc. Este colectivo desarrolló una amplia actividad intelectual en el Reino Unido, participando en diversas instituciones culturales y empresas educativas como el Ateneo Español o la Universidad de Londres.

Pero si hay que explicar la marcha de los estudios económicos en España, señala Fuentes Quintana, es imprescindible tener presentes su larga decadencia en la segunda mitad del siglo XIX (con su empirismo y abandono del estudio) y su despertar a comienzos del XX con la "generación del 98" (Antonio Flores de Lemus, José Zumalacárregui y Francisco Bernís) cuando se vuelve a conectar con el pensamiento económico vigente para explicar los problemas españoles y buscar soluciones.

Flores de Lemus representa al alto funcionario o consejero, inmune a la política de manera que ni el paso de la monarquía parlamentaria a la dictadura ni de ahí a la República supusieron que dejara de servir al Estado, algo que solamente consiguió la Guerra Civil pues marchó en 1936 a Francia de donde no regresó hasta 1939, cuando se la aparta de la cátedra que había alcanzado para, posteriormente, ser denunciado por su escasa afección al nuevo régimen (al igual que otros 17 catedráticos) dada su actuación durante la contienda y sus previos. En 1940 fue denunciado ante el Tribunal de Responsabilidades Políticas y posteriormente absuelto en 1945, cuatro años después de su muerte. Fue repuesto en el escalafón de catedráticos a título póstumo en 1982. Flores emprendió en solitario la tarea de reformar la Hacienda y toda la vida económica española como jefe de la sección estadística del Ministerio de Hacienda, lo que no frenó, su curiosidad intelectual sino que la impulsó y le hizo llegar a la econometría con los adelantados y desde allí observar al avance de Keynes, cuya Teoría general encajaba muy bien con su línea de evolución doctrinal. Pero como ya advirtió el propio Zumalacárregui "en España no hubo un ambiente propicio para la ciencia económica", por eso Flores, pese a adelantarse a las principales teorías de su época, no tuvo proyección más allá de su círculo más próximo.

Zumalacárregui consideraba la economía como una ciencia humana. Los actos humanos, dice, no pueden confundirse con hechos de orden material, pero la regularidad y la constancia de los acontecimientos "morales", evidenciados por la estadística de los actos humanos, es compatible con la libertad individual. Por su parte, Bernís trata de explicar el insuficiente desarrollo económico español y lo hace en base a la pérdida del imperio colonial de ultramar y al carácter dependiente del desarrollo español, dado que España se centra en el comercio y en una agricultura insuficientemente desarrollada. 
Por todo esto si la historia de Menéndez Pelayo se publicase hoy sus volúmenes se verían notablemente aumentados: en el siglo XX español son numerosos los no incluidos en los libros de historia ni en los libros de texto, los no reconocidos en ningún ámbito público. Y es que si algún acontecimiento es crucial para explicar en la España del siglo XX el desarrollo de las Ciencias Sociales es el exilio de muchos de sus intelectuales a raíz de la Guerra Civil española entre 1936, cuando la idea del pueblo que lucha por su independencia volvería a mostrar su vitalidad al producirse la gran confrontación armada entre unas posiciones políticas polarizadas en dos bandos. Tanto los republicanos como los nacionales recurrieron por igual a la retórica reivindicativa y dolida de la "agresión extranjera" al manifestar que la guerra no era una guerra civil sino una nueva guerra nacional, una lucha más por la supervivencia, por ser fieles a nosotros mismos. España se defendía ahora según la versión republicana contra Hitler y Mussolini y según la versión del bando "nacional" que resultaría vencedor contra la conjura judeo-masónica-marxista orquestada por Moscú.

En este sentido, Elías Díaz sostiene que una historia del pensamiento español posterior a 1939 tiene siempre que comenzar, forzosamente, con el recuerdo del exilio de una buena parte de nuestros principales intelectuales en torno a esos años y los pensadores exiliados, que eran quienes permitían seguir en conexión con la cultura europea de raíz liberal y lejos del pensamiento franquista, conservador y circunscrito al pensamiento reaccionario.

\section{II}

Durante el segundo exilio fueron muchos quienes marcharon a América. Entre ellos hay muchos economistas, de los que un poco más adelante hablaremos, pero también filósofos, juristas, sociólogos, etc. Es imprescindible nombrar a Francisco de Ayala, exilado primero en Argentina, luego a Puerto Rico y los Estados Unidos y, ya jubilado, de nuevo a España, ya durante la democracia. Para Ayala el intelectual debe ocupar un espacio público, intervenir en los debates y comprender y hacer comprender lo que en el mundo ocurre. Para ello ha de trazar mapas que expliquen el presente y los procesos que a él han contribuido. Por esta labor, precisamente, es por lo que hay que incluir su figura como precursor, aunque haya sido desde el exterior, de las ciencias sociales en general y la sociología en particular.

También entre los sociólogos merece la pena resaltar la figura de José Medina Echavarría (1903-1977), que ocupó una cátedra de Filosofía del Derecho en la Universidad de Murcia, temática en ese momento muy próxima a la inquietud sociológica, y fue letrado de las Cortes pasando durante la Guerra Civil a ser el máximo representante de España en Polonia. Medina, traductor de Max Weber y posiblemente el más importante sociólogo en lengua castellana, ha sido un perfecto desconocido en los ambientes sociológicos españoles y su primer libro se publica en España después de su muerte. Sin embargo, instalado en Chile tras su exilio, en América Latina siempre fue aceptado como alguien relevante, con categoría magistral, por las dos principales corrientes que se han disputado y dividido la sociología latinoamericana: la Sociología Científica o de la Sociología de la Modernización y la Sociología "comprometida" y crítica de la anterior, preocupada por el estudio de la estructura social y que Gunder Frank dio a conocer como "teoría de la dependencia", que han adecuado la sociología europea y la norteamericana al medio socio-cultural en que tenían que desenvolverse, ofreciendo una serie de categorías de análisis y unos temas de interés adecuados a la realidad latinoamericana.

Medina no es encajable en ninguna de las dos corrientes. Él participa de un armazón teórico que muchas veces se ha opuesto a la visión marxista de la sociología (Simmel, Weber, Mannheim, Comte o Spencer). Su modelo social es el propio de los liberales clásicos, 
su paradigma es el de la sociedad liberal, aunque acepte alguna instrumentalización puntual socialista del desarrollo, tema que le preocupa. Para él es la democracia un medio de alcanzar el desarrollo y un fin, algo que le une a la corriente de la sociología de la modernización aunque no considere, como ésta, la sociedad latinoamericana como dual. Pero en común con la teoría de la dependencia tiene su consideración de la sociología como algo no neutro. Fue un defensor de la paz. Vivió exilado y así murió, aunque visitó la "España de la Transición", pero regresó a Chile pese a que en esos momentos gobernaba la dictadura chilena que le parecía tan inaceptable como le había parecido la española.

Este final de exiliado ya voluntario le une a Wenceslao Roces, historiador, político y filósofo más que economista, que ejerció una notable influencia en el pensamiento económico al ser el traductor de El Capital de Marx y participar en la edición de muchos textos marxistas. Perteneciente desde su inicio a la órbita del Partido Comunista de España hubo de exilarse primero en 1935 y tras su vuelta, con el triunfo del Frente Popular en 1936, hubo de volver al exilio en 1939 con la derrota de la Segunda República. Vivió en México, donde fue profesor de Derecho en la Universidad Autónoma de México y regresó a España en 1977, cuando se presentó, en junio de ese año, al Senado en las primeras elecciones democráticas tras la dictadura franquista pero, pese a conseguir su escaño, regresó en noviembre de ese mismo año a México donde murió en 1992 habiendo alcanzado el mayor reconocimiento. También Indalecio Prieto, que ocupó como ministro distintos ministerios durante la República, escribió en numerosos diarios estando exilado en México.

$\mathrm{Y}$ es que Latinoamérica en general, y México en particular, fueron los grandes receptores de todos los exilados españoles. En Argentina se instalaron muchos de quienes estaban ligados al Derecho, en Puerto Rico y Estados Unidos los ligados a la Historia. Pero en México universidades como la Universidad Autónoma de México y organismos como la Comisión Económica para América Latina (CEPAL) en Chile, los acogieron y se beneficiaron de sus aportaciones. La pérdida neta fue para España.

Muchos de los exilados fueron economistas y los exilados en México tenían en común su contribución desde diferentes ámbitos al análisis y al debate de los problemas económicos y sus teorías. También compartían la influencia del pensamiento inglés, concretamente del keynesiano, y también del marxismo. Ambas influencias afectaron a la firme defensa de la intervención del Estado tanto en el ámbito de lo económico como de lo social y político que todos ellos realizan pero que, curiosamente, en la práctica se tradujo en la mayor parte de los casos en la defensa de una intervención estatal mucho menor que la practicada por la autarquía franquista en España. Es decir, la mayoría de ellos defendieron un sistema liberal y progresista que daba a los Estados y a las sociedades un papel clave en el desarrollo de las instituciones y de los factores asociados a la producción.

$\mathrm{Si}$ hemos de destacar algunas ideas principales de este grupo de economistas podemos comenzar con la consideración de que el bienestar general es el objetivo de toda economía y de toda sociedad fundada en el principio de la democracia. Por eso, para ellos, el elemento de análisis y medición de todas las magnitudes económicas debe ser la tasa de crecimiento del salario real y consideran que la creación monetaria y el crédito bancario no tienen otro respaldo que el de la producción.

Uno de estos economistas, Antonio Sacristán Colás, creía que la creación monetaria convertida en una actividad lucrativa se convierte en un costo para la sociedad y en una caída en las funciones básicas del Estado soberano. Así, en un libro titulado "Keynes ante la crisis mundial de los años ochenta", publicado en 1985, creía que la crisis y la paralización de las 
economías deudoras estaba causada no por el déficit presupuestario al que se le imputó sino en la drástica elevación de las tasas de interés por lo que las políticas del Fondo Monetario Internacional sin contrarrestar la inflación acentuaron la crisis. En relación al Fondo Monetario Internacional otro de estos economistas, Alfredo Lagunilla Iñárritu, mantenía que el Fondo, gobernado por el voto de los países acreedores y con monedas duras no ofrecía soluciones reales a los deudores.

También la influencia del pensamiento inglés puede observarse en Javier Marqués (que hizo el postgrado en la London School of Economics) y que, ante la posible creación de la Unión Aduanera de América Latina, afirmaba que una unión aduanera o económica cualquiera beneficia o daña a nacionales que no son los consumidores sino los capitalistas, que son quienes realmente tienen influencia política. De ahí que resalta la importancia de plantearse qué debe ser primero, si la unión política o la económica. En el mismo sentido Cristóbal Lara Beautell en 1970 expresó su convicción de que para la integración y la unión regional que suponía la creación de un Mercado Común Centroamericano era absolutamente necesario el apoyo político.

Muchos de ellos se preocuparon por los problemas y procesos de la agricultura y la planeación sectorial ya que conciben la agricultura como una condición indispensable para el desarrollo económico global. Así por ejemplo, entre los economistas exiliados en Latinoamérica consideraban que el atraso de su economía venía dado por el estrangulamiento agrícola y le daban al Estado un importante papel en la concesión de los créditos agrícolas y en la administración de subsidios necesarios para una producción creciente y estable para compensar la escasa incorporación tecnológica, el régimen de tenencia del suelo $\mathrm{u}$ la deficiencia en el proceso inversor y su financiamiento.

Y puesto que hemos mencionado la guerra civil como causa de este segundo exilio, es necesario recoger algunas de las reflexiones que en este grupo suscitan las consecuencias económicas y sociales de la misma. Es Manuel Sánchez Sarto el que, partiendo de que las guerras las financian los pueblos, vencedores y vencidos, propone imaginar qué sería si todo el gasto militar destinado a la destrucción y la muerte de la I Guerra Mundial (que considera responsable del poderío económico de los Estados Unidos, la conformación del bloque soviético y la guerra fría) fuese destinado al bienestar mundial. Este mismo autor, en el ámbito de influencia liberal y progresista que señalamos, consideraba en 1945 que las bases para el pleno empleo eran un consumo sostenido, salarios y precios razonablemente estables y movilidad geográfica y ocupacional de los trabajadores.

\section{III}

Para finalizar, quizá deberíamos relacionar estos procesos de exclusión y de expulsión con el proceso de modernización de España y su salida del Antiguo Régimen que, si como hemos visto se materializa en el siglo XIX, se inicia mucho antes, a finales del siglo $\mathrm{XV}$ con la aventura americana.

Fue con la forja del Imperio colonial en América como España se convierte en la mayor potencia del mundo occidental. La apertura española fue la apertura europea, pero a pesar de ser la primera no fue la española una apertura hecha bajo el signo de la modernidad. Como explica Antonio Miguel Bernal, el hecho es que se ocupa América de la misma manera en que se habían ido ocupando los territorios conquistados a los moros hasta ese momento, es decir, repartiendo prebendas y territorios, adelantando fronteras, etc. La construcción del Estado español va unida a la forja del Imperio aunque, a diferencia de lo que hacían otros estados europeos emergentes en los que su Imperio quedaba sujeto a las necesidades del Estado, aquí fue España la que acabó subordinada, al menos en términos 
económicos, a las colonias. Con ello el proceso de apertura y modernización quedó a medio hacer. Y eso, como vimos, cuando se encara el siglo XIX y cuando durante el mismo se pierden todas las colonias, supuso un reacción defensiva y paralizante que dejó ese proceso a expensas, la mayor parte del tiempo, de los elementos más ligados a la conservación del pasado y más antimodernizantes.

En ese contexto, que también marca el siglo XX, pobre papel tienen los pensadores y los científicos que se sitúan (o son situados) las más de las veces en los márgenes de la sociedad y el Estado, cuando no los abandonan ellos mismos en su búsqueda del saber y el progreso, ejes de la modernización pero que aquí son mirados con recelo por la reacción e ignorados generalmente por todos los responsables políticos. Al fin y al cabo, en una sociedad como la que hemos esbozado escaso papel tienen intelectuales y científicos, sobre todo los que no son "orgánicos". Y esto refuerza la tendencia al retraso respecto al resto de los países desarrollados.

Podemos decir así que si bien España es un país europeo en realidad está fuera de Europa como muestra su importante tendencia hacia la polarización que, como hemos visto, hace que en su historia abunden sobre todo las guerras internas, civiles o no, y apenas las internacionales (salvo las que mantuvo con Perú y EEUU en el siglo XIX) como sucede en el resto de los países modernos.

\section{BIBLIOGRAFÍA}

ÁLVAREZ JUNCO, J. (2003): Mater dolorosa. La idea de España en el siglo XIX, Madrid, Taurus. BERNAL, A. M. (2006): España, proyecto inacabado. Costes/beneficios del Imperio, Madrid, Marcial Pons.

CORREA, E. y GIRÓN, A. (2009): Economistas españoles del exilio en México, Madrid, Agencia Española de Cooperación Internacional para el Desarrollo, Ministerio de Asuntos - Exteriores y Cooperación.

FUENTES QUINTANA, E. (2001): Economía y economistas españoles, 6, Barcelona, Galaxia Gutenberg-Círculo de Lectores.

MAESTRE ALFONSO, J. (1991): José Medina Echavarría. Introducción, Madrid, Ediciones de Cultura Hispánica, ICI.

MUNÁRRIZ PERALTA, J. (1967): En defensa de las Cortes, Introducción, Madrid, Ciencia Nueva. RIBES LEIVA, A. J. (2006): "La mirada sociológica y el compromiso con el presente de Francisco Ayala" en Ayala, F. Miradas sobre el presente: ensayos de sociología, Madrid, Fundación Santander Central Hispano.

\section{Breve currículo:}

\section{Adela Roldán Márquez.}

Licenciada en Ciencias Políticas y Sociología por la Universidad Complutense de Madrid y Doctora en Sociología por la Universidad Rey Juan Carlos (Madrid). En la actualidad es Profesora Titular de Universidad adscrita al Departamento de Sociología en la Universidad de Sevilla.

\section{Myriam González Limón.}

Licenciada en Derecho y Ciencias Económicas por la Universidad de Sevilla. Doctora en Economía por la misma universidad. Actualmente es Profesora Colaboradora en el Departamento de Análisis Económico y Economía Política de la misma universidad. 